\title{
Gas-Forming Intracerebral Abscess with Pediatric Parafalcine Subdural Empyema with Review of Literature
}

\author{
Sharad Pandey ${ }^{1}$ Vivek Sharma ${ }^{1}$ Kulwant Singh ${ }^{1}$ Anurag Sahu ${ }^{1}$ Deepa Pandey ${ }^{2}$ \\ ${ }^{1}$ Department of Neurosurgery, Sir Sunder Lal Hospital, Institute of Medical \\ Sciences, Banaras Hindu University, Varanasi, Uttar Pradesh, India \\ 2 Department of Clinical Microbiology, DLW, Varanasi, Uttar Pradesh, India \\ Address for correspondence Sharad Pandey, MS, MCh, Department \\ of Neurosurgery, Sir Sunder Lal Hospital, Institute of Medical \\ Sciences, Banaras Hindu University, Varanasi, Uttar Pradesh 221005, \\ India (e-mail: drsharad23@yahoo.com).
}

Indian J Neurosurg 2015;4:164-172.
Abstract
Keywords
- gas-forming intracerebral abscess
- subdural empyema
- chronic suppurative otitis media

\begin{abstract}
A brain abscess is a focal, intracerebral infection that begins as a localized area of cerebritis and develops into a collection of pus surrounded by a well-vascularized capsule. The incidence of brain abscess varies from 1 to $2 \%$ of all intracranial space occupying lesions in developed countries to $8 \%$ in the developing countries. We reported a case of an 8-year-old girl who presented with gas-forming intracerebral abscess with parafalcine subdural empyema as a complication of bilateral chronic suppurative otitis media with coalescent mastoiditis and reviewed the literature.
\end{abstract}

\section{Introduction}

Despite the advent of modern neurosurgical techniques, new antibiotics, and new powerful imaging technologies, brain abscess remains a potentially fatal central nervous system infection. ${ }^{1}$ A brain abscess is a focal, intracerebral infection that begins as a localized area of cerebritis and develops into a collection of pus surrounded by a wellvascularized capsule. ${ }^{2}$ It results from direct extension of a cranial infection (e.g., subdural empyema, osteomyelitis, mastoiditis, and sinusitis), penetrating head wounds, or hematogenous spread (e.g., in bacterial endocarditis, bronchiectasis, congenital heart disease with right-to-left shunt, or intravenous (IV) drug abuse. ${ }^{3}$ Subdural empyema (before 1943, it was called subdural abscess) is a suppurative infection that forms in the subdural space, which has no anatomic barrier to spread. It should be distinguished from abscess which forms within brain substance, surrounded by tissue reaction with fibrin and collagen capsule formation. ${ }^{4}$ Overall, 20 to $25 \%$ of imaging studies showed that subdural empyema may be complicated by cerebral abscess. ${ }^{4}$ Subdural abscess is rare, occurring more commonly with sinusitis than with otitis media. ${ }^{5}$ Both acute and chronic otitis media are

received

April 10, 2015

accepted after revision

June 26, 2015

published online

November 24, 2015
DOI http://dx.doi.org/ 10.1055/s-0035-1559804. ISSN 2277-954X. considered as serious diseases in children because of their possible extracranial and/or intracranial extension. The incidence of brain abscess varies from 1 to $2 \%$ of all intracranial space occupying lesions in the developed countries to $8 \%$ in the developing countries. ${ }^{6}$ Brain abscesses are associated with otitis media in around $30 \%$ of cases. ${ }^{7}$ It is estimated that only 1 in $2 \times 10^{6}$ episodes of otitis media results in a brain abscess. ${ }^{8}$ Early presumptive clinical diagnosis supported by radiological evidence (computerized axial tomography [CT] scan and magnetic resonance imaging [MRI]) is the mainstay of diagnosis. ${ }^{9}$ In this case, we witnessed a gas-forming brain abscess in the early stage related with chronic mastoiditis where gas formation disappeared in the later phase of the abscess formation.

\section{Case Summary}

An 8-year-old girl presented at our hospital with history of purulent discharge from left ear since 1 year, progressive headache, seizures, and altered sensorium since 2 days. Before presenting at our hospital, patient had taken treatment from a private hospital where her laboratory parameters were hemoglobin $11.4 \mathrm{~g} / \mathrm{dL}$, total leucocyte (c) 2015 Neurological Surgeons' Society
of India

License terms

$\Theta(1) \Theta$ 
count $27,100 / \mathrm{mm}^{3}$, differential leukocyte count was neutrophil $90 \%$, lymphocyte $05 \%$, eosinophil $03 \%$, monocyte $02 \%$, basophil $0 \%$, erythrocyte sedimentation rate (ESR) $26 \mathrm{~mm} / \mathrm{h}$, and platelet count $3,10,000 / \mathrm{mm}^{3}$. Contrastenhanced computed tomography (CECT) head showed left temporal lobe abscess with air ( - Fig. 1). Four days later, her hematological profile was hemoglobin $11.6 \mathrm{~g} / \mathrm{dL}$, total leukocyte count $24,400 / \mathrm{mm}^{3}$, differential leukocyte count was neutrophil $83 \%$, lymphocyte $10 \%$, eosinophil $06 \%$, monocyte $01 \%$, basophil $0 \%$, and ESR $32 \mathrm{~mm} / \mathrm{h}$. Blood culture revealed Staphylococcus aureus which was sensitive to oxacillin, trimethoprim-sulphamethoxazole (1.25/23.75), erythromycin, and clindamycin. She was started on IV antibiotic therapy, details of which were not available. One week later, repeat CECT was done, which showed hypodense lesion measuring $28 \times 20 \mathrm{~mm}$ with thick enhancing wall and air in the left temporal lobe region with parafalcine abscess (-Fig. 2). She was continued with IV antibiotics as told by patient's attendant. Approximately 2 weeks later, her condition deteriorated and she presented in the neurosurgery emergency with dizziness and altered sensorium. At the time of admission, her laboratory parameters were hemoglobin $9.6 \mathrm{~g} / \mathrm{dL}$, total leucocyte count $7,200 / \mathrm{mm}^{3}$, and differential leukocyte count was neutrophil $57 \%$, lymphocyte $39 \%$, eosinophil $03 \%$, monocyte $01 \%$, basophil 0\%, ESR $26 \mathrm{~mm} / \mathrm{h}$, platelet count 2,04,000/ $\mathrm{mm}^{3}$. CECT head was performed which showed a large round, intraaxial ring-enhancing lesion measuring approximately $4.8 \times 3.8 \mathrm{~cm}$ in left parietotemporal region with moderate surrounding vasogenic edema (grade 2 ) with midline shift of $8 \mathrm{~mm}$ to right side (subfalcine herniation), subdural, extraaxial, hypodense area with peripheral enhancement noted in the parasagittal region of parietotemporal lobe suggestive of subdural empyema, and partial fluid to soft tissue density opacification noted in the bilateral middle air cavity and mastoid air cells suggestive of chronic suppurative otitis media (CSOM) with coalescent mastoiditis (-Fig. 3). Surgical intervention was planned and left temporal craniotomy with abscess aspiration and excision of abscess cavity was undertaken. Through a left posterior parietal burr hole, parafalcine abscess was also aspirated. Thick yellow pus was evacuated which was sent for microbiological examination. Direct Gram staining of the pus from abscess cavity showed plenty of pus cells with no bacterial or fungal elements. Bacteriological culture was sterile after 48 hours of incubation. She was empirically started on combination of IV antibiotics (viz, ampicillin-cloxacillin, gentamycin, and metronidazole.) The patient neither developed any postoperative complications nor fever. Contrast CT taken 3 weeks postoperatively showed intracranial hypodense fluid attenuating collection measuring $1.5 \times 1.6 \times 1.5 \mathrm{~cm}$ with fluid debris in the left temporal region (-Fig. 4). Patient showed remarkable improvement and underwent cortical mastoidectomy for the treatment of primary cause.

\section{Discussion and Review of Literature}

Otogenic brain abscess usually occurs in the temporal lobe and cerebellum (2:1). Nearly all otogenic brain abscesses have underlying chronic suppurative otorrhea. More than half of all otogenic brain abscesses have an associated cholesteatoma. Although the relative incidence of complications related to chronic otitis media is debated within the literature, many authors agree that the most dangerous complication is intracranial abscess formation which carries a 40 to $50 \%$ mortality rate. ${ }^{10}$ In otogenic brain abscesses, the routine procedures are conservative or radical mastoidectomy, drainage of the abscess, incision of the sigmoid sinus and removal from its lumen of the clot, and broad-spectrum IV antibiotics therapy. ${ }^{5}$

Treatment of brain abscess is still a subject of controversy. ${ }^{11}$ Treatment requires a multidisciplinary approach (-Fig. 5).

\section{Role of Radiological Evaluation}

Besides good clinical history and examination, radiological evaluation helps a lot in patient management. Suspicion of intracranial complication demands radiologic evaluation in

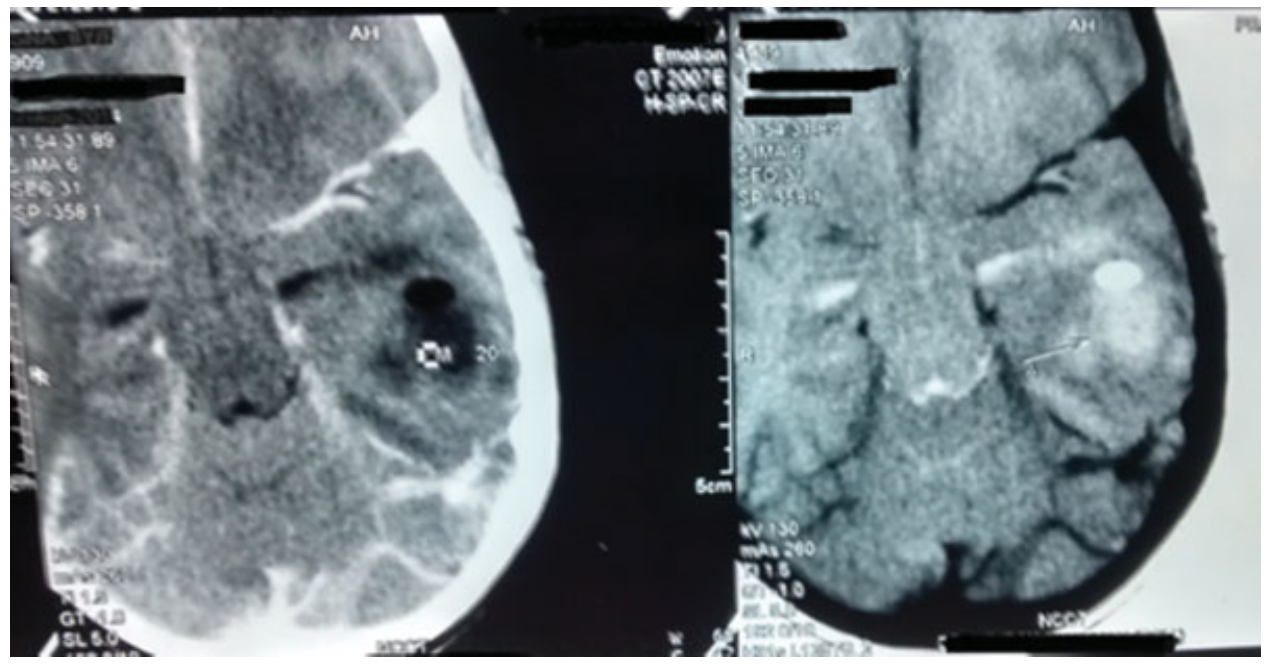

Fig. 1 Contrast-enhanced computed tomography head showing left temporal lobe abscess with air. 


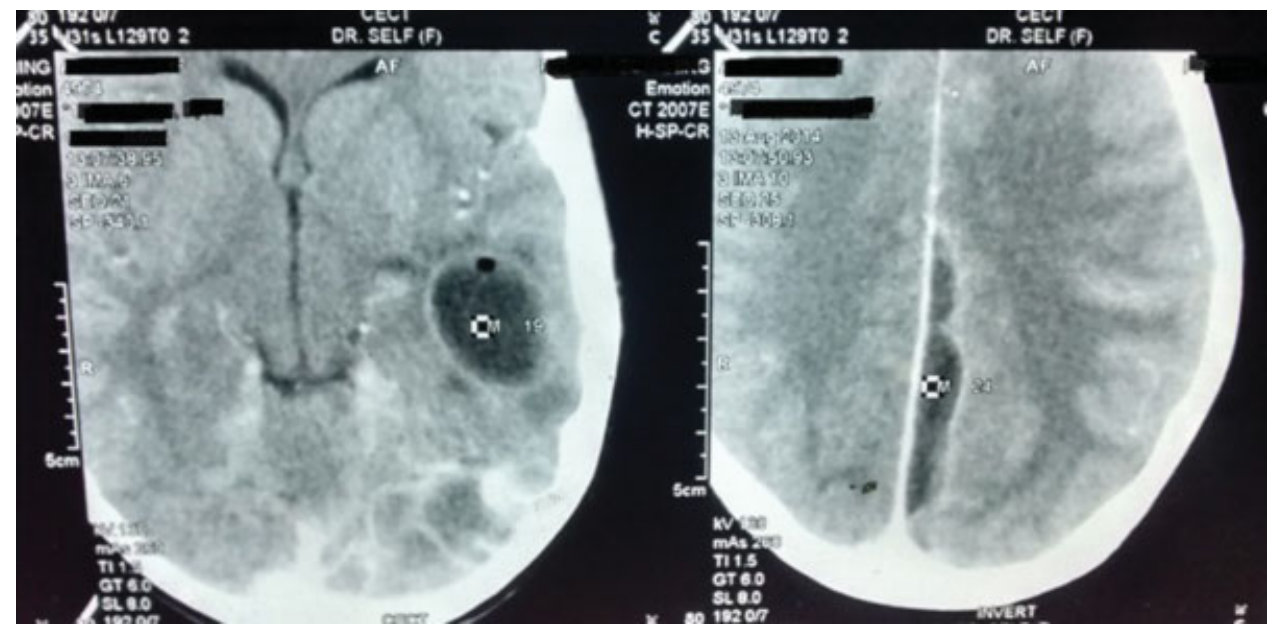

Fig. 2 Contrast-enhanced computed tomography showing hypodense lesion with enhancing wall and air in the left temporal lobe region with parafalcine abscess.

patients with active chronic otitis media which is crucial to diagnosis and management. Axial CT with contrast is the usual modality of choice preferably with thin cuts, which can demonstrate the presence or absence of an abscess as well as details regarding its size and location. ${ }^{10}$

This patient had initial CT finding suggestive of temporal lobe abscess which later on progressed thus showing parietotemporal lobe abscess with parafalcine abscess in the parasagittal region of parietotemporal lobe.

CT scan as a diagnostic tool helps in early diagnosis, planning the treatment modality and its efficacy, and sequential follow-up. It helps in detection, exact localization, and accurate characterization, determination of number, size, stage of the abscess, associated hydrocephalus, raised intracranial pressure (ICP), edema and associated infection, pathology such as subdural empyema, ventriculitis, multiple abscesses, and loculations, and to some extent the nature of pus, thus helps in treatment planning. ${ }^{6,12}$ Also, CT is excellent for detecting predisposing factors for brain abscess, such as sinusitis, mastoiditis, and soft tissue infections. ${ }^{12}$ Our patient gave history of unilateral ear discharge; however, CT scan revealed bilateral CSOM with coalescent mastoiditis.

The classic appearance of brain abscess on contrastenhanced CT scan is lesion having a smooth, thin, regular wall with a decreased density both in the center of the lesion (representing pyogenic material) and in the surrounding white matter (representing edema). The abscess wall may be isodense or denser than the brain on noncontrast CT scan. ${ }^{11}$ MRI is more sensitive than CT scan as it can detect abscess in the earliest stages besides providing better anatomic details, multiple imaging planes, lack of bony artifacts, especially in posterior fossa, and increased sensitivity for follow-up examination. ${ }^{13,14}$

Other improved radio diagnostic noninvasive techniques
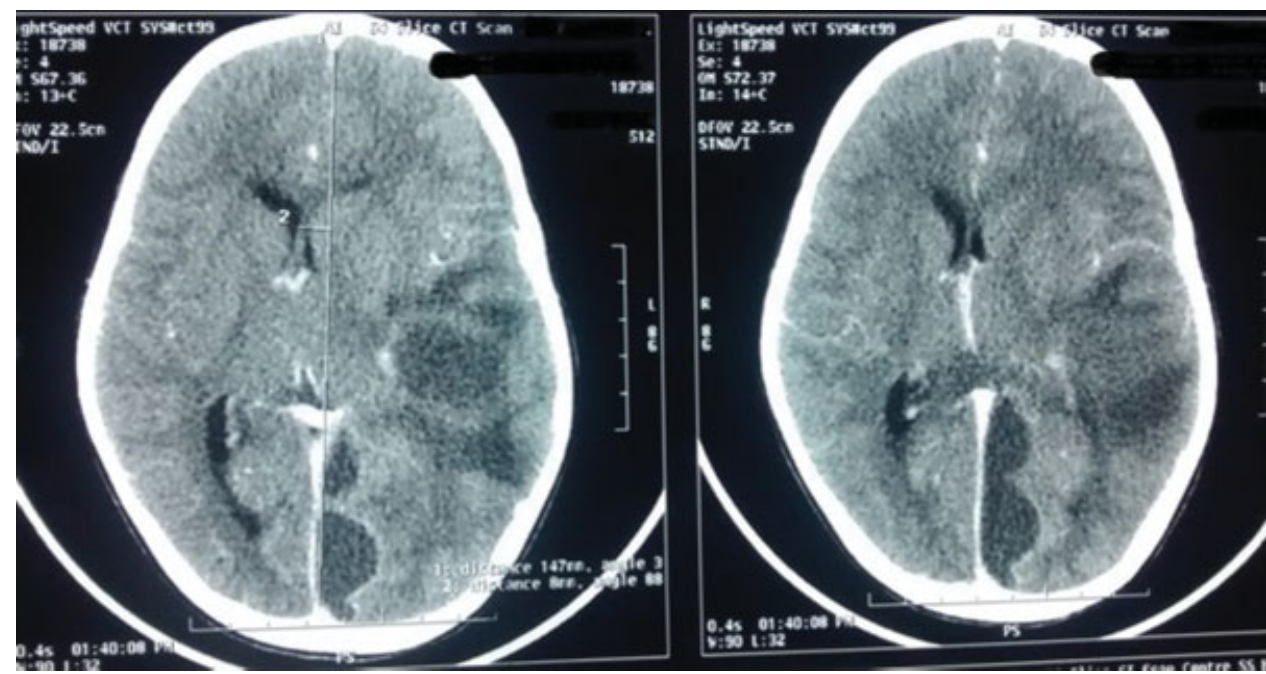

Fig. 3 Contrast-enhanced computed tomography head showing a round, intra-axial ring enhancing lesion in left parietotemporal region with midline shift with subdural empyema. 

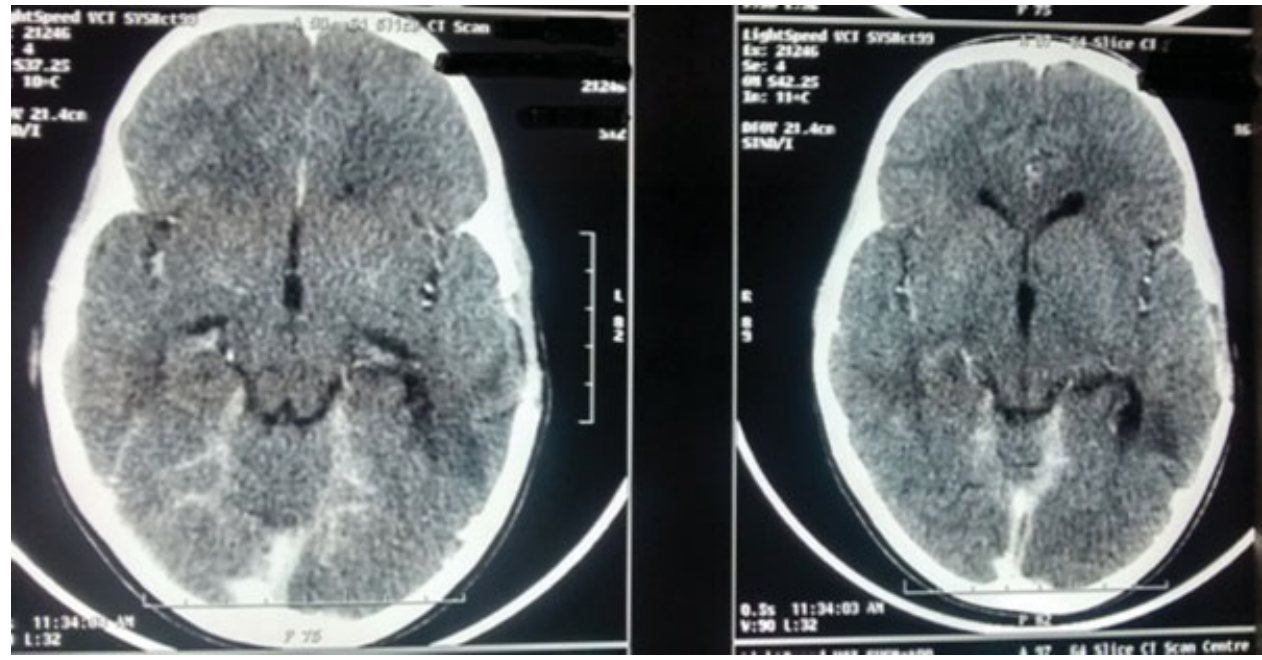

Fig. 4 Postoperative contrast-enhanced computed tomography showing intracranial hypodense fluid debris in the left temporal region.

available to detect brain abscess are magnetic resonance spectroscopy and diffusion-weighted imaging which also helps to differentiate abscess from necrotic or cystic tumors. $^{15,16}$

\section{Pathogenesis of Brain abscess}

Brain abscess pathology can be divided into four stages (-Table 1). In this case, we witnessed a gas-forming brain abscess in the early stage related with chronic mastoiditis where gas formation disappeared in the later phase of the abscess formation. Presence of intracranial gas can be attributed to two reasons, first being penetration of gas through an abnormal communication between the exterior and intracranium, and second because of the bacterial fermentation leading to gas formation. ${ }^{7}$ Radiological imaging suggested that the presence of intracranial gas was because of the bacterial fermentation, as no abnormal communication between the exterior and intracranium was seen in CT scans. Also, the hematological profile showed leukocytosis with neutrophilia indicating the presence of bacterial infection.

Causative microorganism of gas forming brain abscess include Clostridium perfringens, Escherichia coli, Klebsiella pneumoniae, Enterobacter, Proteus, Pseudomonas, Peptostreptococcus, and Bacteroides spp. ${ }^{17}$ Infection by gas-forming organisms mostly occur in patients with

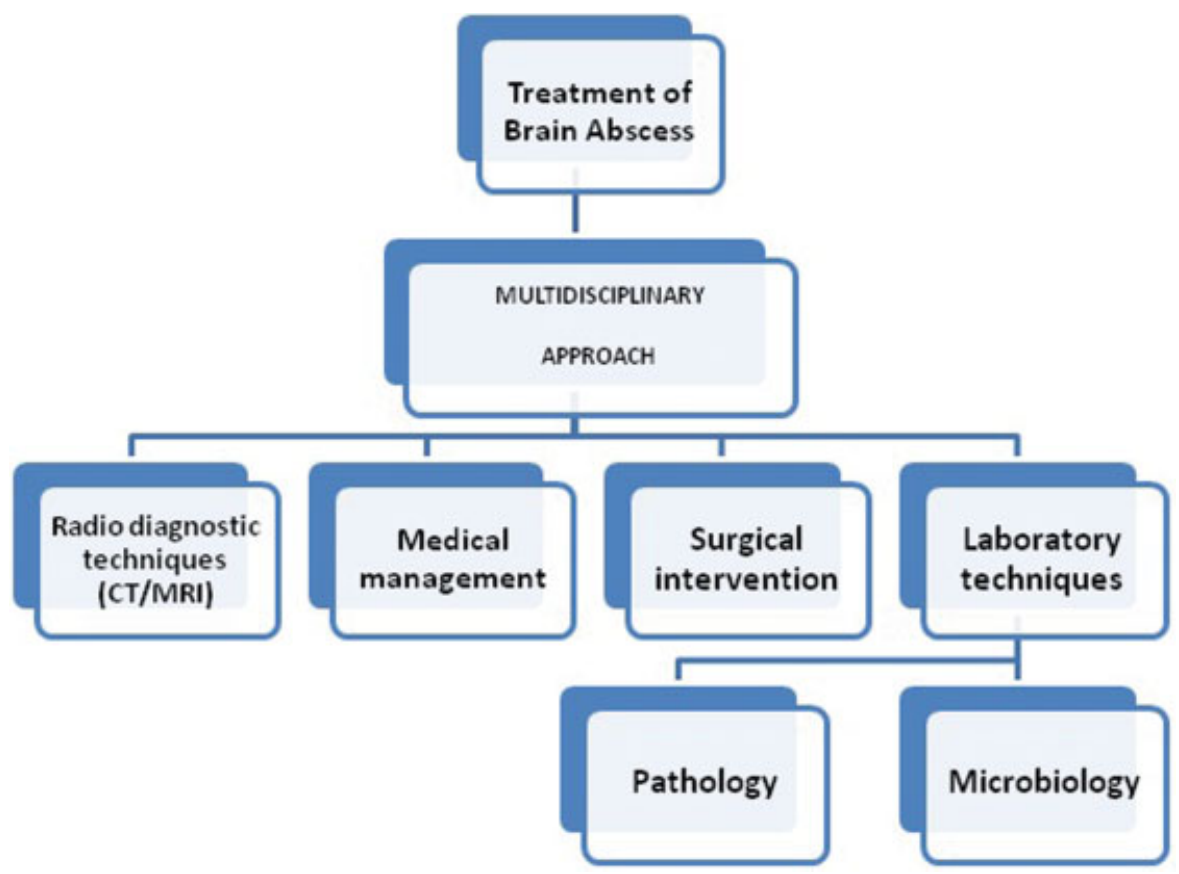

Fig. 5 Multidisciplinary approach in management of brain abscess. CT, computerized tomography; MRI, magnetic resonance imaging. 
Table 1 Stages of brain abscess

\begin{tabular}{|l|l|l|}
\hline Stage & Time & Characteristics \\
\hline Early cerebritis & $1-4 \mathrm{~d}$ & Neutrophil accumulation, tissue necrosis, and edema \\
\hline Late cerebritis & $4-10 \mathrm{~d}$ & Predominant macrophage and lymphocytic infiltrate \\
\hline Early capsule formation & $11-14 \mathrm{~d}$ & $\begin{array}{l}\text { Formation of well-vascularized abscess wall which is } \\
\text { crucial in sequestering the lesion, maintaining integrity of the } \\
\text { brain function, and limiting expansion of infective process }\end{array}$ \\
\hline Late capsule formation & $>14 \mathrm{~d}$ & At 3-4 wk abscess cavity becomes thick and is amenable to excision \\
\hline
\end{tabular}

predisposing factors, such as trauma, sepsis, paranasal sepsis, and otitis media. Also, the source of primary infection helps to assess the most likely microorganism/ microorganisms involved in brain abscess (-Table 2 ) and thus deciding the empirical antimicrobial treatment.

Interestingly, Taguchi et al reported a case of gascontaining brain abscess due to Fusobacterium nucleatum. ${ }^{17}$ Heilpern and Lorber reported that aerobic gram-negative bacilli are isolated in 23 to 33\% of cases, usually in mixed culture, with Proteus spp. and Pseudomonas aeruginosa being frequent isolates from abscess of otogenic origin. ${ }^{18}$ Liliang et al reported 15 patients with Klebsiella brain abscess with relatively high mortality rate. ${ }^{19}$ of 15 patients, 2 (13\%) were found to have gas-forming abscess. Cho and Park described the first case to show the gasforming brain abscess with intraventricular empyema caused by Klebsiella pneumoniae. ${ }^{20}$ Liliang et $\mathrm{al}^{21}$ and Rau et $\mathrm{al}^{22}$ pointed out that when brain abscess occurs in diabetic patients or has a gas-forming appearance, Klebsiella pneumoniae infection should be considered and further attention should be paid to discover whether other metastatic septic abscess exist and early diagnosis and treatment are essential for survival. Van Leer et al described a case of brain abscesses with gas formation following otitis media, where several microorganisms, including Clostridium glycolicum, were cultured and was the first report of an infection in an immunocompetent patient associated with this microorganism. ${ }^{7}$ They concluded that Clostridium glycolicum may act as a copathogen in infections in immunocompetent individuals. One of the largest reported case series by Nathoo et al found that majority of patients undergoing surgery (53.2\%) had isolation of a single organism, the gram-positive organisms being the most common, Staphylococcus aureus and Staphylococcus epidermidis, especially in traumatic brain abscess (BA). Streptococcus milleri isolates were predominant

Table 2 Commonly isolated microorganisms depending on the predisposing factor

\begin{tabular}{|l|l|}
\hline Site of infection & Most likely microorganism \\
\hline Paranasal sinusitis & $\begin{array}{l}\text { Microaerophilic and anaerobic streptococci, Haemophilus spp., } \\
\text { Bacteroides spp. (nonfragilis), and Fusobacterium spp. }\end{array}$ \\
\hline Otitis media mastoiditis & $\begin{array}{l}\text { Bacteroides (including fragilis spp.), Pseudomonas aeruginosa, } \\
\text { Enterobacteriaceae, Streptococcus, Morganella morganii, fungi } \\
\text { like Aspergillus }\end{array}$ \\
\hline Dental infections & Streptococcus and gram-negative bacilli, Bacteroides fragilis \\
\hline Endocarditis & Streptococci viridans, Staphylococcus aureus \\
\hline Congenital cyanotic heart disease & Streptococci \\
\hline Intra-abdominal or pelvic infection & $\begin{array}{l}\text { Anaerobic Bacteroides, Prevotella melaninogenica, } \\
\text { Propionibacterium fusobacterium, Actinomyces }\end{array}$ \\
\hline Pyogenic lung infection & Streptococcus spp., Actinomyces spp., Fusobcterium spp. \\
\hline Urinary tract infection & Enterobacteriaceae and Pseudomonadaceae \\
\hline Penetrating head trauma and neurosurgical procedures & Staphylococcus aureus \\
\hline Immigrants & $\begin{array}{l}\text { Parasites-Taenia, Entamoeba, Schistosoma, Paragonimus, } \\
\text { Echinococcus }\end{array}$ \\
\hline Immunocompromised & $\begin{array}{l}\text { Toxoplasma gondii, Nocardia asteroides, Cryptococcus neoformans, } \\
\text { Mycobacterium, Listeria monocytogenes }\end{array}$ \\
\hline Neutropenia & $\begin{array}{l}\text { Aspergillus spp., Candida spp., Mucor, Enterobacteriaceae, } \\
\text { Pseudomonas aeruginosa }\end{array}$ \\
\hline
\end{tabular}


in patients with paranasal sinusitis, whereas Proteus mirabilis was most commonly isolated in otogenic infections. ${ }^{23}$ Using $16 S$ ribosomal DNA amplification, Al Masalma et al achieved pathogen identification in 9 of 21 (43\%) culture-negative BA, identified 44 distinct bacterial species not previously described in BA, and determined polymicrobial infections at a significantly higher rate than by culture. $^{24}$

\section{Medical Management}

The indications for medical management are depicted in -Fig. 6.

Aforementioned criteria help in determining the line of management; however, each case has to be individualized ${ }^{25}$ and treated accordingly. We decided to continue our patient with medical line of management comprising of combination antimicrobials, antiepileptics, and symptomatic medication. It is important to acknowledge the fact that the physiology of the blood-brain barrier and the blood-cerebrospinal fluid (CSF) barrier varies and not all antimicrobial drugs have good penetration from the systemic circulation into brain tissue. The antimicrobial concentrations in plasma cannot be used to predict the concentrations of these agents in brain tissue or intracranial pus as the penetration of drugs into CSF differs from that into brain tissue or intracranial pus. Initial therapy should include broad spectrum antibiotics which can penetrate blood-brain and blood-CSF barriers in adequate concentrations providing anaerobic coverage, such as, a third-generation cephalosporin and metronidazole. Vancomycin is added if there is a history of penetrating trauma or a recent neurosurgical procedure. As per Rehman et al, chloramphenicol, an antimicrobial agent, is used rarely today in the United States because of its associated adverse effects, is a broad-spectrum agent that is highly effective against many gram-positive and gram-negative bacteria, spirochetes, chlamydiae, and rickettsia. Because of its ability to achieve high concentrations in the cerebrospinal fluid, it is advised as a second-line therapy for culture negative brain abscesses. ${ }^{1}$

Song et al recommended that the effectiveness of tertiary generation cephalosporin + vancomycin + metronidazole for bacterial brain abscess was $88 \%$ and use of combination antibiotics in cases with sterile culture report. ${ }^{26}$ We empirically started our patient on IV ampicillin-cloxacillin, gentamycin, metronidazole and had to continue these for 3 weeks as the pus drained was found to be bacteriologically sterile after 48 hours of incubation. Antibiotics such as penicillin, ampicillin, cefuroxime, chloramphenicol, cotrimoxazole, ceftazidime, and metronidazole achieve therapeutic concentrations in intracranial pus, and have been administered successfully as treatment in various

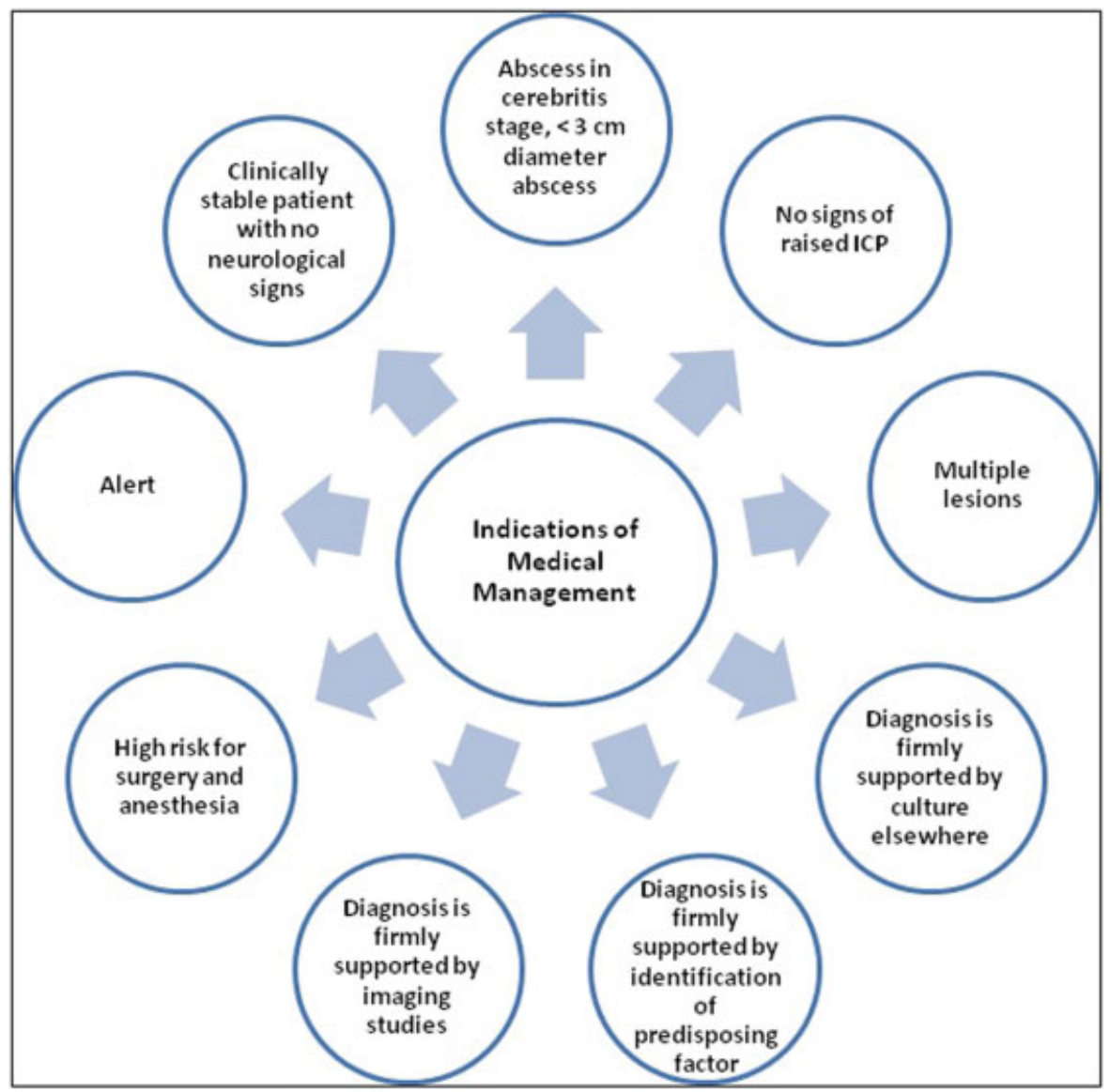

Fig. 6 Indications of medical management. ICP, intracranial pressure. 
combinations. ${ }^{27}$ Arlotti et al recommends collecting sample from a brain abscess before starting antibiotic therapy or, at least within not more than 3 days of the start of therapy. ${ }^{28}$ In this case, patient had already received IV antibiotics for approximately 3 weeks before presenting to us which could be one of the reasons for culture being sterile. One week later, we decided to undertake left temporal craniotomy with aspiration of abscess and excision of abscess cavity. The guiding principles ${ }^{27}$ for surgical management by "Infection in Neurosurgery Working Party of the British Society for Antimicrobial Chemotherapy," are shown in -Fig. 7.

Besides diagnosis, surgical intervention also helps to remove as much as purulent material as possible thus improving the chances of recovery. Surgery should also be considered if there is any clinical deterioration, significant mass effect, neurological deficit, multiple lesions in surgically accessible locations, doubtful diagnosis, presumably resistant organisms, ${ }^{25}$ and multiple loculations. In most instances, aspiration of the purulent material is sufficient to initiate the healing of abscess. However, surgical excision becomes mandatory if the pus is thick and in multiloculated abscesses. Urgent evacuation of abscess is required for subdural empyema and cerebellar abscess.

Different therapeutic approaches include craniotomy with primary excision, burr hole and aspiration, stereotactic aspiration, neuronavigation aspiration, ultrasound-guided aspiration, endoscopic aspiration, and stereotactic endoscopic aspiration. Various surgical comparison studies have been conducted comparing various methods of treatment. Mut et al compared the efficacies of aspiration of the BA cavity versus excision of BA capsule and their impact on postoperative antibiotic use and the length of hospital stay. They reported three residual/ recurrence cases in the aspiration group, who needed a second aspiration, whereas no residual/recurrence was observed in the excision group. Postoperative use of antibiotics and the length of hospital stay for IV antibiotic administration were significantly lesser in the excision group. $^{29}$ In another study by $\mathrm{Su}$ et al, excision was found to be more appropriate surgical choice in multiloculated abscess. $^{30}$ Similar were the conclusions by Sarmast et al comparing the efficacies of aspiration versus excision for the management of large solitary encapsulated pyogenic BA located in superficial noneloquent areas. ${ }^{31}$ Another study by Tan et al compared the burr hole approach versus craniotomy used as treatment for superficial BA and its outcome and found that patients who had undergone craniotomy and excision of abscess showed a significantly earlier improvement in neurological function, better radiological clearance, and lower rate of resurgery as compared with the burr hole aspiration group $(p<0.05)$, but with no significant difference between the two surgical methods regarding to neurological improvement at 3 months, morbidity, and mortality. ${ }^{32}$

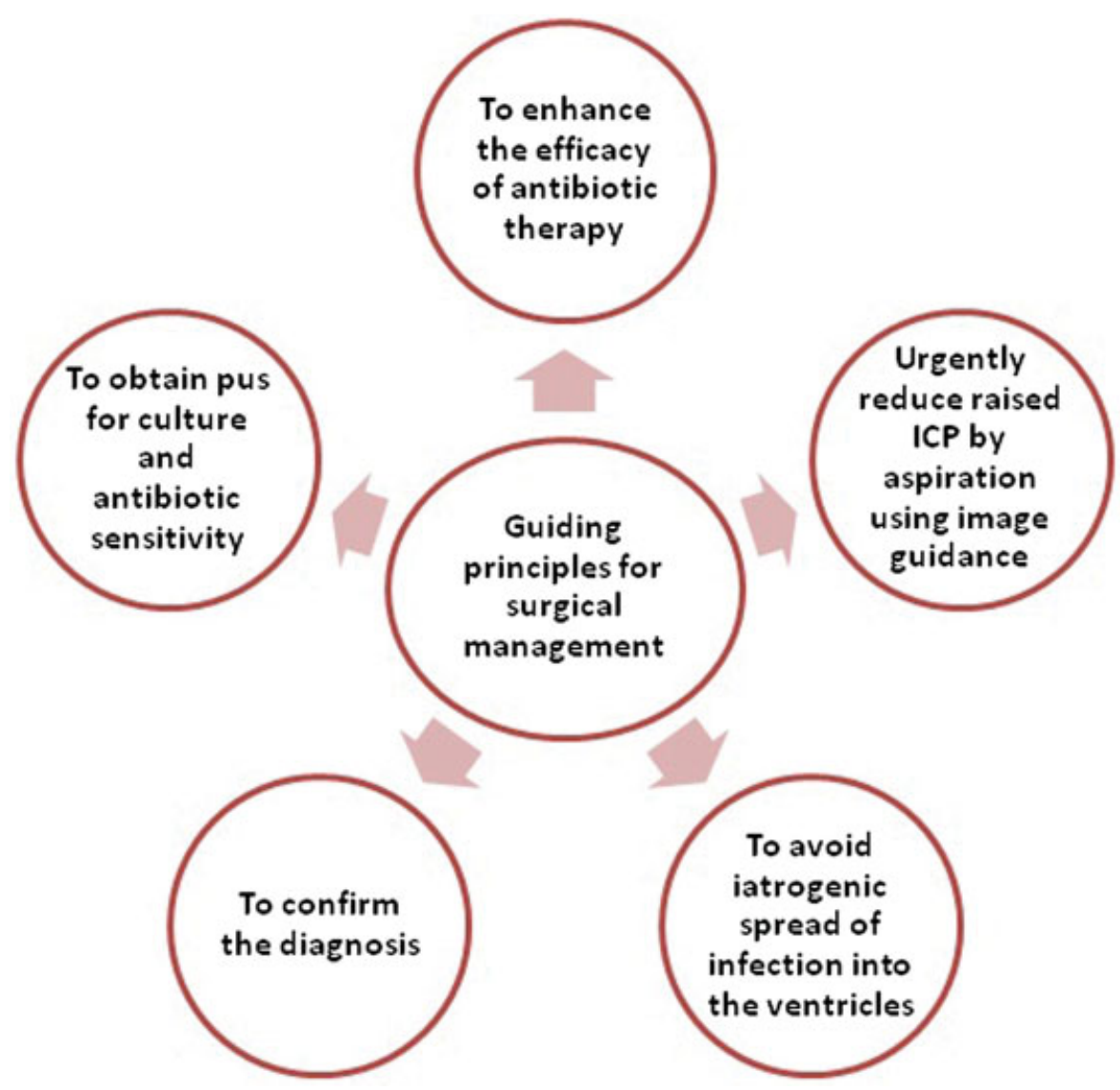

Fig. 7 Guidelines for surgical management. 
Various studies conducted, till date, were not designed in a manner so as to provide strong conclusions regarding the surgical technique. However, aspiration of brain abscess is preferred nowadays as it is safe, rapid procedure, can be performed under local anesthesia (except in children). ${ }^{12}$ It provides rapid relief from raised ICP for single or multiple, deep- and/or "eloquent area"located lesions. Disadvantages of aspiration are needed for repeated aspirations and high risk of iatrogenic puncture of the ventricle and subarachnoid leakage of pus leading to meningitis and/or ventriculitis. ${ }^{33}$

\section{Parafalcine Subdural Empyemas}

Till date, very few cases of parafalcine subdural empyemas have been reported in children. We reviewed the literature and found only five articles related to parafalcine subdural empyemas in children. In a study by Pathak et al, of 41 cases with subdural empyema, only 4 patients had parafalcine empyema. ${ }^{34}$ Another study, conducted by Banerjee et al reported 65 pediatric patients with supratentorial subdural empyemas. $^{35}$ Niklewski et al witnessed two cases of pediatric parafalcine empyemas as a complication of a sinusitis frontalis. ${ }^{36}$

Parafalcine empyemas can present variably with symptoms being quite nonspecific like fever and headache or specific such as hemiparesis, impairment of consciousness, and seizures. Most of the pediatric brain abscesses generally present with headache (48\%), fever (48\%), nausea-vomiting (36\%), and seizures (29.3\%). ${ }^{37}$ In our patient, although symptoms such as fever, headache, and seizures were present but no cranial nerve palsy or hemiparesis was found.

In our study, aspiration of the parafalcine abscess was done and broad spectrum IV antibiotics were continued for 3 weeks. The patient responded well and repeat CECT head after 3 weeks showed resolution of parafalcine abscess. For early diagnosis, a contrast-enhanced CT scan and a nonenhanced scan are essential, but the procedure of choice is an MRI with contrast. This was quite in contrast to the case study by Niklewski where in spite of antibiotic therapy and surgery with drainage of the abscess, the symptoms reappeared and the abscess increased as seen in the follow-up CT and MRI and a second surgery, despite sufficient antibiotic treatment was undertaken.

\section{Conclusion}

To conclude, an early diagnosis, aspiration of pus, good laboratory support, and broad spectrum antibiotic therapy is very helpful in curtailing the patient morbidity, mortality, duration of hospital stay, health care expenses, and resources. Thus, "an action in time saves nine" is a suitable proverb in this scenario.

\section{Conflict of Interest}

None.

\section{References}

1 Rehman AU, Rehman T, Ali R. Multi-antibiotic resistant brain abscess sensitive only to chloramphenicol: a case report. Cases J 2009;2:6352

2 Moorthy RK, Rajshekhar V. Management of brain abscess: an overview. Neurosurg Focus 2008;24(6):E3

3 Tunkel AR, Wispelwey B, Scheld MW. Brain abscess. In: Mandell GL, Douglas RG, Bennett JE, eds. Principles and Practice of Infectious Diseases, 5th ed. New York, NY: Churchill Livingstone; 2000:1016-1028

4 Greenberg MS. Infections. In: Handbook of Neurosurgery. 7th ed. New York, NY: Thieme Publisher; 2010:342-393

5 Kuczkowski J, Mikaszewski B. Intracranial complications of acute and chronic mastoiditis: report of two cases in children. Int J Pediatr Otorhinolaryngol 2001;60(3):227-237

6 Muzumdar D, Jhawar S, Goel A. Brain abscess: an overview. Int J Surg 2011;9(2):136-144

7 Van Leer C, Wensing AMJ, van Leeuwen JP, Zandbergen EGJ, Swanink CMA. Clostridium glycolicum isolated from a patient with otogenic brain abscesses. J Clin Microbiol 2009;47(2): 511-513

8 Leskinen K, Jero J. Acute complications of otitis media in adults. Clin Otolaryngol 2005;30(6):511-516

9 Mathisen GE, Johnson JP. Brain abscess. Clin Infect Dis 1997; 25(4):763-779, quiz 780-781

10 Neuroradiology on the net. Gas producing otogenic brain abscess with cerebral edema and pseudosubarachnoid hemorrhage sign. 2009. Available at: http://neuroradiologyonthenet.blogspot.in/ 2009/03/gas-producing-otogenic-brain-abscess.html. Accessed July 21, 2015

11 Rosenblum ML, Mampalam TJ, Pons VG. Controversies in the management of brain abscesses. Clin Neurosurg 1986; 33:603-632

12 Dharker SR, Sardana VR, Purohit D. Brain Abscess. In: Ramamurthi R, Tandon PN, eds. Text Book of Neurosurgery, 3rd ed. New Delhi, India: Jaypee Brothers Medical Publishers; 2012:695-707

13 Adachi J, Uki J, Kazumoto K, Takeda F. Diagnosis of brainstem abscess in the cerebritis stage by magnetic resonance imagingcase report. Neurol Med Chir (Tokyo) 1995;35(7):467-470

14 Sze G, Zimmerman RD. The magnetic resonance imaging of infections and inflammatory diseases. Radiol Clin North Am 1988;26(4):839-859

$15 \mathrm{Kim} \mathrm{SH}$, Chang KH, Song IC, et al. Brain abscess and brain tumor: discrimination with in vivo H-1 MR spectroscopy. Radiology 1997;204(1):239-245

16 Ebisu T, Tanaka C, Umeda M, et al. Discrimination of brain abscess from necrotic or cystic tumors by diffusion-weighted echo planar imaging. Magn Reson Imaging 1996;14(9):1113-1116

17 Taguchi Y, Sato J, Nakamura N. Gas-containing brain abscess due to Fusobacterium nucleatum. Surg Neurol 1981;16(6):408-410

18 Heilpern KL, Lorber B. Focal intracranial infections. Infect Dis Clin North Am 1996;10(4):879-898

19 Liliang PC, Hung KS, Cheng CH, Chen HJ, Ohta I, Lui CC. Rapid gasforming brain abscess due to Klebsiella pneumoniae. Case illustration. J Neurosurg 1999;91(6):1060

20 Cho KT, Park BJ. Gas-forming brain abscess caused by Klebsiella pneumoniae. J Korean Neurosurg Soc 2008;44(6):382-384

21 Liliang PC, Lin YC, Su TM, et al. Klebsiella brain abscess in adults. Infection 2001;29(2):81-86

22 Rau CS, Chang WN, Lin YC, et al. Brain abscess caused by aerobic Gram-negative bacilli: clinical features and therapeutic outcomes. Clin Neurol Neurosurg 2002;105(1):60-65

23 Nathoo N, Nadvi SS, Narotam PK, van Dellen JR. Brain abscess: management and outcome analysis of a computed tomography era experience with 973 patients. World Neurosurg 2011;75(56):716-726, discussion 612-617 
24 Al Masalma M, Lonjon M, Richet $\mathrm{H}$, et al. Metagenomic analysis of brain abscesses identifies specific bacterial associations. Clin Infect Dis 2012;54(2):202-210

25 Sharma BS, Gupta SK, Khosla VK. Current concepts in the management of pyogenic brain abscess. Neurol India 2000; 48(2):105-111

26 Song L, Guo F, Zhang W, et al. Clinical features and outcome analysis of 90 cases with brain abscess in central China. Neurol Sci 2008;29(6):425-430

27 Infection in Neurosurgery Working Party of the British Society for Antimicrobial Chemotherapy. The rational use of antibiotics in the treatment of brain abscess. Br J Neurosurg 2000;14(6):525-530

28 Arlotti M, Grossi P, Pea F, et al; GISIG (Gruppo Italiano di Studio sulle Infezioni Gravi) Working Group on Brain Abscesses. Consensus document on controversial issues for the treatment of infections of the central nervous system: bacterial brain abscesses. Int J Infect Dis 2010;14(Suppl 4):S79-S92

29 Mut M, Hazer B, Narin F, Akalan N, Ozgen T. Aspiration or capsule excision? Analysis of treatment results for brain abscesses at single institute. Turk Neurosurg 2009;19(1):36-41

30 Su TM, Lan CM, Tsai YD, Lee TC, Lu CH, Chang WN. Multiloculated pyogenic brain abscess: experience in 25 patients. Neurosurgery 2008;62(Suppl 2):556-561
31 Sarmast AH, Showkat HI, Kirmani AR, et al. Aspiration versus excision: a single center experience of forty-seven patients with brain abscess over 10 years. Neurol Med Chir (Tokyo) 2012; 52(10):724-730

32 Tan WM, Adnan JS, Mohamad Haspani MS. Treatment outcome of superficial cerebral abscess: an analysis of two surgical methods. Malays J Med Sci 2010;17(1):23-29

33 Hall WA, Truwit CL. The surgical management of infections involving the cerebrum. Neurosurgery 2008;62(Suppl 2):519-530, discussion 530-531

34 Pathak A, Sharma BS, Mathuriya SN, Khosla VK, Khandelwal N, Kak VK. Controversies in the management of subdural empyema. A study of 41 cases with review of literature. Acta Neurochir (Wien) 1990;102(1-2):25-32

35 Banerjee AD, Pandey P, Devi BI, Sampath S, Chandramouli BA. Pediatric supratentorial subdural empyemas: a retrospective analysis of 65 cases. Pediatr Neurosurg 2009; 45(1):11-18

36 Niklewski F, Petridis AK, Hourani JA, et al. Pediatric parafalcine empyemas. J Surg Case Rep 2013;doi: 10.1093/jscr/ rjt067

37 Ozsürekci Y, Kara A, Cengiz AB, et al. Brain abscess in childhood: a 28-year experience. Turk J Pediatr 2012;54(2):144-149 